\title{
Strategies for the production of high-content fructo-oligosaccharides through the removal of small saccharides by co-culture or successive fermentation with yeast
}

\author{
C. Nobre ${ }^{\mathrm{a}, \mathrm{b}, *}$, C.C. Castro ${ }^{\mathrm{b}}$, A.-L. Hantson ${ }^{\mathrm{b}}$, J.A. Teixeira ${ }^{\mathrm{a}}$, G. De Weireld ${ }^{\mathrm{b}}$, L.R. Rodrigues ${ }^{\mathrm{a}}$ \\ a Centre of Biological Engineering, University of Minho, Campus de Gualtar, 4710-057 Braga, Portugal \\ ${ }^{\mathrm{b}}$ Faculté Polytechnique de Mons, Université de Mons, 20 Place du Parc, 7000 Mons, Belgium
}

\section{A R T I C L E I N F O}

\section{Article history:}

Received 25 May 2015

Received in revised form 25 August 2015

Accepted 26 August 2015

Available online 31 August 2015

\section{Keywords:}

Fructo-oligosaccharides

Aureobasidium pullulans

Co-culture

Successive fermentation

Saccharomyces cerevisiae

\begin{abstract}
A B S T R A C T
Fructo-oligosaccharides (FOS) obtained by fermentation of sucrose may be purified at large-scale by continuous chromatography (Simulated Moving Bed: SMB). In order to improve the efficiency of the subsequent SMB purification, the optimization of the fermentative broth composition in salts and sugars was investigated. Fermentations conducted at reduced amount of salts, using Aureobasidium pullulans whole cells, yielded $0.63 \pm 0.03 \mathrm{~g}$ of FOS per gram of initial sucrose. Additionally, a microbial treatment was proposed to reduce the amount of small saccharides in the mixture. Two approaches were evaluated, namely a co-culture of A. pullulans with Saccharomyces cerevisiae; and a two-step fermentation in which FOS were first synthesized by A. pullulans and then the small saccharides were metabolized by S. cerevisiae. Assays were performed in $100 \mathrm{~mL}$ shaken flasks and further scaled-up to a $3 \mathrm{~L}$ working volume bioreactor. Fermentations in two-step were found to be more efficient than the co-culture ones. FOS were obtained with a purity of $81.6 \pm 0.8 \%(\mathrm{w} / \mathrm{w})$, on a dry weight basis, after the second-step fermentation with $S$. cerevisiae. The sucrose amount was reduced from 13.5 to $5.4 \%$ in total sugars, which suggests that FOS from this culture broth will be more efficiently separated by SMB.
\end{abstract}

(C) 2015 Published by Elsevier Ltd.

\section{Introduction}

The consumers' interest in healthy and high nutritional food has significantly increased in the recent years. This growing trend towards the adoption of healthier lifestyles has been the main driver for the great demand of functional ingredients in the global food market, such as fructo-oligosaccharides (FOS) which exhibit prebiotic activity (Preter, Hamer, Windey, \& Verbeke, 2011). Industrially, FOS are produced from sucrose through transfructosylation by enzymes with a maximum theoretical yield between 0.55 and $0.60 \mathrm{~g}_{\mathrm{FOS}} \mathrm{g}_{\text {Sucrose }}{ }^{-1}$ (Nishizawa, Nakajima, \& Nabetani, 2001; Sangeetha, Ramesh, \& Prapulla, 2005). Recently, a slightly higher yield, 0.64 g $_{\text {FOS }}$ g $_{\text {Sucrose }}{ }^{-1}$, was obtained in a fermentation conducted with whole cells of Aureobasidium pullulans instead of the

\footnotetext{
* Corresponding author at: Centre of Biological Engineering, University of Minho, Campus de Gualtar, 4710-057 Braga, Portugal.

E-mail addresses: clarissenobre@deb.uminho.pt, clarissenobre@gmail.com (C. Nobre), Cristiana.Castro@umons.ac.be (C.C. Castro),

Anne-Lise.Hantson@umons.ac.be (A.-L. Hantson), jateixeira@deb.uminho.pt (J.A. Teixeira), Guy.DeWeireld@umons.ac.be (G. De Weireld), lrmr@deb.uminho.pt (L.R. Rodrigues).
}

enzymes (Dominguez et al., 2012). One of the biggest challenges in FOS production is achieving high yields of oligosaccharides since glucose is also produced during the fermentation inhibiting the fructosyl-transferring reaction. Besides, FOS are hydrolysed during the enzymatic synthesis (Yun \& Song, 1993). Thus, some unreacted residual sucrose is always present in the fermentation mixture. The presence of small saccharides in the final fermentation mixture (fructose, glucose and sucrose) decreases the prebiotic activity of the mixture. Therefore, a downstream removal step is needed to enable the incorporation of these FOS mixtures in diabetic, dietetic and healthy foods.

Several techniques have been studied to remove small sugars from the FOS mixtures including ultra and nanofiltration (Pinelo, Jonsson, \& Meyer, 2009), activated charcoal systems (Nobre, Teixeira, \& Rodrigues, 2012), microbial treatment (Crittenden \& Playne, 2002) and ion-exchange chromatography (Nobre, Suvarov, \& De Weireld, 2014). The purification of FOS is not straightforward due to the physicochemical similarities between the different oligosaccharides and the smaller saccharides. Hence, to obtain purified FOS in large-scale with minimal product losses, the combination of two methods can be envisaged, namely a microbial treatment followed by continuous chromatography separation (Simulated Moving Bed: SMB). The impact of the continuous 
removal of glucose and residual sucrose from the medium during the FOS synthesis to achieve higher fermentation yields has been attempted (Jung, Kim, Jeon, \& Lee, 1993; Sheu, Lio, Chen, Lin, \& Duan, 2001; Sheu, Duan, Cheng, Bi, \& Chen, 2002; Sheu, Chang, Wang, Wu, \& Huang, 2013; Yun, Lee, \& Song, 1994). Most studies involve using a mixture of enzymes, one responsible for the FOS synthesis and the other able to convert small sugars without FOS hydrolysis activity (Jung et al., 1993; Lin \& Lee, 2008; Sheu et al., 2001; Yang, Wang, Teng, \& Zhang, 2008; Yun et al., 1994).

The aim of this work was to optimize the fermentative broth composition in salts and small sugars to enable its further feeding into a SMB plant for the FOS purification. A. pullulans (FOS producer) and Saccharomyces cerevisiae (able to ferment glucose, fructose and sucrose to ethanol) were used. The pre-purification phase was performed either in one-step fermentation (co-culture of both strains) or in two-step fermentation, where firstly the FOS mixture was bioproduced by A. pullulans and secondly, the small saccharides from the FOS mixture were metabolized by $S$. cerevisiae.

\section{Materials and methods}

\subsection{Microorganisms and culture conditions}

The fungus A. pullulans CCY 27-1-94 was grown in pellets form, which facilitates the biomass harvesting from the culture broth. The strain was maintained on Petri plates containing Czapeck Dox Agar (Oxoid, UK) medium at $4{ }^{\circ} \mathrm{C}$ and was subcultured every month. A concentrated spore suspension was prepared by placing the fungus in plates at $28^{\circ} \mathrm{C}$ for 5 days. At that time, spores were scraped from the plates with a $0.1 \%(\mathrm{w} / \mathrm{v})$ solution of Tween 80 (Panreac, AppliChem, Spain). The number of spores in the concentrated solution was determined by counting in a microscope using an improved Neubauer chamber. Afterwards, the suspension was diluted to a $9 \times 10^{7}$ spores $\mathrm{mL}^{-1}$.

S. cerevisiae 11982 was grown in YEG (yeast extract-glucose) culture medium, previously autoclaved at $121^{\circ} \mathrm{C}$ for $20 \mathrm{~min}$, containing $5 \mathrm{~g} \mathrm{~L}^{-1}$ yeast extract and $20 \mathrm{~g} \mathrm{~L}^{-1}$ glucose (both from Fluka, Germany), for $24 \mathrm{~h}$, at $30^{\circ} \mathrm{C}$ and $150 \mathrm{rpm}$ of agitation. The strain was transferred monthly to fresh YEG agar plates and stored at $4{ }^{\circ} \mathrm{C}$ after incubation at $30^{\circ} \mathrm{C}$ for 5 days.

\subsection{FOS production}

\subsubsection{Experimental design}

The composition of the fermentation medium for A. pullulans growth and FOS synthesis was firstly optimized regarding its salt composition. The optimal concentration levels of both $\mathrm{NaNO}_{3}$ and $\mathrm{KH}_{2} \mathrm{PO}_{4}$ salts were determined using an experimental design. The Response Surface Method (RSM) was determined with a threelevel factorial design and two factors, for the maximization of FOS production. High, intermediate and low levels of salts concentration were considered: $5.0,12.5$ and $20.0 \mathrm{~g} \mathrm{~L}^{-1}$ for $\mathrm{NaNO}_{3}$ and $4.0,6.0$ and $8.0 \mathrm{~g} \mathrm{~L}^{-1}$ for $\mathrm{KH}_{2} \mathrm{PO}_{4}$. A total of 11 independent experiments, including three replicates at the central point of the design were conducted. Positive effects were considered significant for $p$-values lower than 0.05 (Haaland, 1989). The statistical experimental design was generated and evaluated using the JMP ${ }^{\mathrm{TM}}$, version 10 - The Statistical Discovery Software, from SAS.

\subsubsection{Shaken flask fermentations}

Shaken flasks of $100 \mathrm{~mL}$ with test tube aluminium caps were used. An aliquot of $1 \mathrm{~mL}$ of $A$. pullulans spores suspension $\left(9 \times 10^{7}\right.$ spores $\left.\mathrm{mL}^{-1}\right)$ was transferred to $50 \mathrm{~mL}$ of fermentation medium, containing: $200 \mathrm{~g} \mathrm{~L}^{-1}$ sucrose, $0.5 \mathrm{~g} \mathrm{~L}^{-1} \mathrm{KCl}, 0.35 \mathrm{~g} \mathrm{~L}^{-1}$ $\mathrm{K}_{2} \mathrm{SO}_{4}, 0.5 \mathrm{gL}^{-1} \mathrm{MgSO}_{4} \cdot 7 \mathrm{H}_{2} \mathrm{O}, 0.01 \mathrm{gL}^{-1} \mathrm{FeSO}_{4} .7 \mathrm{H}_{2} \mathrm{O}$ and the respective optimum concentrations of $\mathrm{NaNO}_{3}$ and $\mathrm{KH}_{2} \mathrm{PO}_{4}$ previously determined by RSM (Section 2.2.1). All salts were purchased from VWR (Belgium). Chemicals used were of analytical grade, except for the sucrose used for FOS synthesis, which was a commercial sugar obtained by Raffinerie Tirlemontoise, S.A., Belgium. Culture medium was previously autoclaved at $121^{\circ} \mathrm{C}$ for $15 \mathrm{~min}$. Sucrose and $\mathrm{FeSO}_{4} \cdot 7 \mathrm{H}_{2} \mathrm{O}$ solutions were sterilized by filtration $(0.2 \mu \mathrm{m})$.

The operational conditions and fermentative broth composition were adapted from Dominguez et al. (2012). The $\mathrm{pH}$ of the culture medium was adjusted to 5.5 before inoculation and the fermentations were performed at $32{ }^{\circ} \mathrm{C}$ with an agitation of $150 \mathrm{rpm}$. Several samples were taken at different time points to further determine the sugars profile.

\subsubsection{Bioreactor fermentation}

An aliquot of $1 \mathrm{~mL}$ of $A$. pullulans spores suspension $\left(9 \times 10^{7}\right.$ spores $\left.\mathrm{mL}^{-1}\right)$ was transferred to $100 \mathrm{~mL}$ of inoculum medium containing $100 \mathrm{~g} \mathrm{~L}^{-1}$ sucrose and the same optimized salt concentrations previously used in the shaken flask fermentations. The inoculum was grown at $28^{\circ} \mathrm{C}$ and $150 \mathrm{rpm}$ and transferred after 3 days to a $5 \mathrm{~L}$ bioreactor - BIOSTAT ${ }^{\circledR}$ B module (Sartorius, Germany). A working volume of $3 \mathrm{~L}$ of culture medium $\left(200 \mathrm{~g} \mathrm{~L}^{-1}\right.$ sucrose and salt concentrations equal as the ones used in the inoculum) was used. Salts solutions were autoclaved inside the bioreactor, at $121^{\circ} \mathrm{C}$ for $30 \mathrm{~min}$. Sucrose and $\mathrm{FeSO}_{4} \cdot 7 \mathrm{H}_{2} \mathrm{O}$ solutions were sterilized by filtration $(0.2 \mu \mathrm{m})$ and further added to the bioreactor. Fermentations were carried out at $32{ }^{\circ} \mathrm{C}$ and $385 \mathrm{rpm}$ with a fixed $\mathrm{pH}$ of $5.50 \pm 0.05$.

\subsection{Removal of the small saccharides}

The ability of $S$. cerevisiae to remove mono- and disaccharides from the fermentation broth was evaluated using two different strategies, as illustrated in Fig. 1.

In the one-step fermentation, the fungus A. pullulans and S. cerevisiae were inoculated in the same shaken flask, as a co-culture, for a simultaneous synthesis and purification of FOS through the consumption of the non-oligosaccharides present in the fermentation mixture.

Shaken flasks of $100 \mathrm{~mL}$ containing $50 \mathrm{~mL}$ of culture medium ( $200 \mathrm{~g} \mathrm{~L}^{-1}$ sucrose with the optimized salt composition) were inoculated with an aliquot of $1 \mathrm{~mL}$ of $A$. pullulans spores suspension $\left(9 \times 10^{7}\right.$ spores $\left.\mathrm{mL}^{-1}\right)$ and $1 \mathrm{~mL}$ of S. cerevisiae (with an optical density at $620 \mathrm{~nm}$ of 1.0 ). Fermentations were carried out at $32{ }^{\circ} \mathrm{C}$ and $150 \mathrm{rpm}$ with an initial $\mathrm{pH}$ of 5.50. Additionally, the same assays were performed in a similar culture medium but using $5 \mathrm{~g} \mathrm{~L}^{-1}$ of yeast extract. Samples were taken along the fermentation at different time points to evaluate the sugars profile.

Bioreactor fermentations were performed using $3 \mathrm{~L}$ of the optimized fermentation medium inoculated with $100 \mathrm{~mL}$ of $A$. pullulans inoculum (grown for 3 days at $28^{\circ} \mathrm{C}$ and $150 \mathrm{rpm}$ ) and $60 \mathrm{~mL}$ of $S$. cerevisiae (with an optical density at $620 \mathrm{~nm}$ of 1.0). Additionally, these fermentations were repeated using a similar culture medium though, with $5 \mathrm{~g} \mathrm{~L}^{-1}$ of yeast extract. Experiments were run at $32{ }^{\circ} \mathrm{C}$, $385 \mathrm{rpm}$ and a constant controlled $\mathrm{pH}$, with ortho-phosphoric acid and ammonia solution, at $5.50 \pm 0.05$.

In the two-step fermentation, FOS were synthesized in a firststep fermentation by A. pullulans, as described in Section 2.2.3 "Bioreactor fermentations". The reaction was stopped at the maximum FOS concentration time point and the biomass was removed by filtration using cellulose acetate filters (VWR, Belgium) with a pore size of $0.2 \mu \mathrm{m}$.

The filtered broth was used for the subsequent fermentation and it was inoculated with S. cerevisiae cells (with an optical density at 


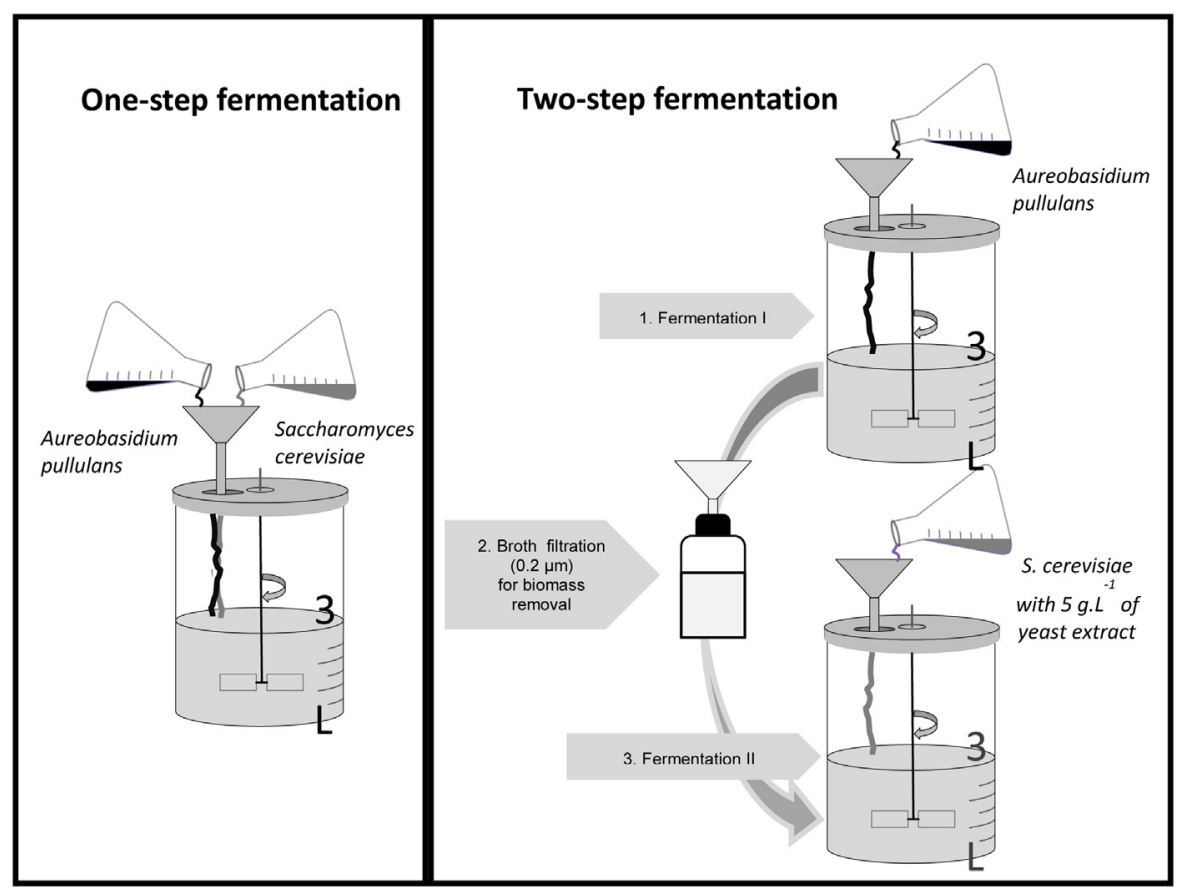

Fig. 1. Strategies for fructo-oligosaccharides purification.

$620 \mathrm{~nm}$ of 1.0 ). In shaken flask experiments, $1 \mathrm{~mL}$ of $S$. cerevisiae cells was used to inoculate $50 \mathrm{~mL}$ of filtered broth, while in the bioreactor $54 \mathrm{~mL}$ of S. cerevisiae cells was used to inoculate $2.7 \mathrm{~L}$. A defined volume of a yeast extract concentrated solution $(1.67 \mathrm{~mL}$ for shaken flaks and $90 \mathrm{~mL}$ for the bioreactor) was added to the second-step fermentation broth in order to obtain a final concentration of $5 \mathrm{~g} \mathrm{~L}^{-1}$. Shaken flask fermentations were carried out at $32^{\circ} \mathrm{C}$ and $150 \mathrm{rpm}$ with an initial $\mathrm{pH}$ of 5.5. The bioreactor fermentations were conducted at $32{ }^{\circ} \mathrm{C}$ and $385 \mathrm{rpm}$ with a constant controlled $\mathrm{pH}$ of $5.50 \pm 0.05$.

\subsection{Sugars and ethanol analysis}

Samples were analyzed by HPLC for sugars and ethanol quantification (Dias et al., 2009; Nobre et al., 2009). A HPLC (Jasco), equipped with a refractive index detector working at $30^{\circ} \mathrm{C}$ and a Prevail Carbohydrate ES $5 \mathrm{u}$ column $(5 \mu \mathrm{m}, 25 \mathrm{~cm} \times 0.46 \mathrm{~cm}$ length $\times$ diameter) (Alltech), was used. A mixture of acetonitrile (HPLC Grade, Carlo Erba, France) in pure-water (70:30 v/v), and $0.04 \%$ of ammonium hydroxide (HPLC Grade, Sigma, Germany) was used as mobile phase. Elution was conducted at $1 \mathrm{~mL} \mathrm{~min}^{-1}$ flow rate and room temperature. The chromatographic signal was recorded and further integrated using the Star Chromatography Workstation software (Varian, USA).

FOS standards, namely 1-kestose $\left(\mathrm{GF}_{2}\right)$, nystose $\left(\mathrm{GF}_{3}\right)$ and 1fructofuranosyl nystose $\left(\mathrm{GF}_{4}\right)$ were acquired from Wako (Japan). Sucrose (GF), fructose (F) and ethanol standards were obtained from Merck (USA) and glucose (G) from VWR (Belgium). All chemicals used for analysis were of analytical grade.

\subsection{Statistical analysis}

Fermentation experiments were carried out in triplicate. Statistical data analysis was performed using analysis of variance (ANOVA) and Tukey's HSD test at a 5\% level of significance.

\section{Results and discussion}

\subsection{FOS production}

The combination of two methods to increase the amount of FOS relative to the other sugars present in mixtures obtained by fermentation with $A$. pullulans was proposed. These methods include a co-culture (one-step) or two-step fermentation, using also a strain able to consume the small sugars, for further improving the SMB chromatography separation.

Since in SMB chromatography cationic resins are used to separate sugars, it is important to reduce the amount of salts in the mixture from which FOS will be purified. Therefore, the effect of decreasing the salt amounts in the culture media used to produce FOS was evaluated. The results obtained for the different initial salt concentrations defined in the experimental design are shown in Table 1 . The fermentation times $47.8 \mathrm{~h}$ and $53.5 \mathrm{~h}$ were selected since the maximum FOS production was obtained around these time points for all the performed fermentations. According to the statistical analysis, neither the concentration of $\mathrm{NaNO}_{3}$ nor of $\mathrm{KH}_{2} \mathrm{PO}_{4}$ affected significantly the FOS production $(p>0.05)$ in the range of concentrations used. The results were evaluated by ANOVA (analysis of variance) and $F$-test at $95 \%$ of confidence level. F-values determined for each model corresponding to maximum FOS concentration, percentage, yield and productivity were $0.90,2.16,0.46$ and 0.11 , respectively. The calculated $F$-values are lower than the listed one $\left(F_{5,5}=5.05\right)$, therefore the differences are considered not statistically significant. In average, the maximum concentration of FOS $\left(101 \pm 3 \mathrm{~g} \mathrm{~L}^{-1}\right)$ was obtained at $51 \pm 2 \mathrm{~h}$ of fermentation, in the shaken flasks. The average fermentation yield was $0.51 \pm 0.01 \mathrm{~g}_{\text {FOS }} \mathrm{g}_{\text {Sucrose }}{ }^{-1}$ with a content of $49 \pm 1 \%$ of FOS in total sugars. These values represent the average of all data obtained in the experimental design.

Fermentations were scaled-up to a $5 \mathrm{~L}$ bioreactor using $5.0 \mathrm{~g} \mathrm{~L}^{-1}$ of $\mathrm{NaNO}_{3}$ and $4.0 \mathrm{~g} \mathrm{~L}^{-1}$ of $\mathrm{KH}_{2} \mathrm{PO}_{4}$. Yields and FOS concentration in the bioreactor (Table 2) were found to be similar to those obtained in previous works using the whole cell of $A$. pullulans at the same 
Table 1

Experimental conditions defined by the experimental design and responses obtained for the optimization of the salt concentrations in the initial fermentation broth.

\begin{tabular}{|c|c|c|c|c|c|c|c|}
\hline & $\mathrm{NaNO}_{3}\left(\mathrm{~g} \mathrm{~L}^{-1}\right)$ & $\mathrm{KH}_{2} \mathrm{PO}_{4}\left(\mathrm{~g} \mathrm{~L}^{-1}\right)$ & Fermentation time $(\mathrm{h})$ & Maximum FOS $\left(\mathrm{g} \mathrm{L}^{-1}\right)$ & $\%$ FOS $(w / w)^{b}$ & Yield ( g Fos $_{\text {Sucrose }}{ }^{-1}$ ) & $Q_{p}\left(\mathrm{~g}_{\mathrm{FOS}} \mathrm{L}^{-1} \mathrm{~h}^{-1}\right)$ \\
\hline A1 & 5.0 & 8.0 & 47.8 & 101.3 & 48.9 & 0.54 & 2.1 \\
\hline A2 & 20.0 & 4.0 & 53.5 & 91.5 & 43.5 & 0.46 & 1.7 \\
\hline $\mathrm{A} 3^{\mathrm{a}}$ & 12.5 & 6.0 & 53.5 & 103.7 & 48.1 & 0.53 & 1.9 \\
\hline A4 & 5.0 & 4.0 & 47.8 & 95.4 & 50.8 & 0.50 & 2.0 \\
\hline A5 & 5.0 & 6.0 & 53.5 & 95.1 & 49.6 & 0.48 & 1.8 \\
\hline A6 & 12.5 & 8.0 & 53.5 & 101.9 & 49.8 & 0.51 & 1.9 \\
\hline$A 7^{\mathrm{a}}$ & 12.5 & 6.0 & 47.8 & 104.3 & 50.6 & 0.53 & 2.2 \\
\hline A8 & 20.0 & 6.0 & 47.8 & 105.7 & 49.3 & 0.53 & 2.2 \\
\hline A9 & 12.5 & 4.0 & 53.5 & 105.6 & 49.7 & 0.53 & 2.0 \\
\hline A10 & 20.0 & 8.0 & 53.5 & 103.0 & 47.0 & 0.52 & 1.9 \\
\hline $\mathrm{A} 11^{\mathrm{a}}$ & 12.5 & 6.0 & 53.5 & 99.9 & 49.0 & 0.50 & 1.9 \\
\hline
\end{tabular}

a Central points; $Q_{p}$ - productivity; FOS - fructo-oligosaccharides.

b On a dry weight basis.

Table 2

Fructo-oligosaccharides (FOS) production by Aureobasidium pullulans using $5.0 \mathrm{~g} \mathrm{~L}^{-1}$ of $\mathrm{NaNO}_{3}$ and $4.0 \mathrm{~g} \mathrm{~L}^{-1}$ of $\mathrm{KH}_{2} \mathrm{PO}_{4}$, in bioreactor.

\begin{tabular}{|c|c|c|c|c|c|c|}
\hline Time $(\mathrm{h})$ & $\operatorname{FOS}\left(\mathrm{g} \mathrm{L}^{-1}\right)$ & $\% \mathrm{FOS}(\mathrm{w} / \mathrm{w})^{\mathrm{a}}$ & $Q_{p}\left(g_{\mathrm{FOS}} \mathrm{L}^{-1} \mathrm{~h}^{-1}\right)$ & Yield ( $\mathrm{g}_{\mathrm{FOS}} \mathrm{g}_{\text {Sucrose }}{ }^{-1}$ ) & Yield ( $\mathrm{g}_{\mathrm{GF} 2} \mathrm{~g}_{\text {Sucrose }^{-1}}$ ) & Yield ( $\mathrm{g}_{\mathrm{GF} 3} \mathrm{~g}_{\text {Sucrose }}{ }^{-1}$ ) \\
\hline 20 & $118.6 \pm 1.6$ & $54.0 \pm 1.6$ & $4.8 \pm 1.4$ & $0.63 \pm 0.03$ & $0.38 \pm 0.08$ & $0.23 \pm 0.05$ \\
\hline
\end{tabular}

$Q_{p}$ - productivity; $\mathrm{GF}_{2}$ - kestose; $\mathrm{GF}_{3}$ - nystose.

a On a dry weight basis.

operational conditions of temperature, $\mathrm{pH}$ and agitation, though using higher concentrations of salts (Dominguez et al., 2012; Nobre et al., 2012). It is noteworthy that in the present work, the time needed to achieve the maximum FOS production was twice as low as in the previous reports, which may be due to a different approach used to prepare the inoculum, thus leading to a process with a much higher productivity $(4.8 \pm 1.4$ compared to the previous $2.9 \mathrm{~g}_{\mathrm{FOS}} \mathrm{L}^{-1} \mathrm{~h}^{-1}$ (Dominguez et al., 2012). The decrease of the $\mathrm{NaNO}_{3}$ amount in the culture from 20.0 to $5.0 \mathrm{~g} \mathrm{~L}^{-1}$ and $\mathrm{KH}_{2} \mathrm{PO}_{4}$ from 7.9 to $4.0 \mathrm{~g} \mathrm{~L}^{-1}$ will also reduce costs associated with the fermentation process, as well as the time associated with the demineralization procedures needed to purify the sugar solution before feed in the SMB plant.

The commercially available FOS are typically produced in twostep processes, in which the enzyme is firstly produced by the microorganism, followed by the extraction and purification of the enzyme, and secondly the fermentation step in which FOS are synthesized (Sangeetha et al., 2005). Fermentations carried out in two-steps, using the A. pullulans crude enzyme, led to a maximum yield production of $0.62 \mathrm{~g}_{\text {Fos }} \mathrm{g}_{\text {Sucrose }}{ }^{-1}$ (Yoshikawa, Amachi, Shinoyama, \& Fujii, 2008). In the present work, a yield of $0.63 \pm 0.03 \mathrm{~g}_{\text {FOS }} \mathrm{g}_{\text {Sucrose }}{ }^{-1}$ was obtained, for one-step fermentation, working with the whole cells, thus resulting in a much less expensive and time consuming process for the production of FOS.

S. cerevisiae was used in the co-culture and two-step fermentations. The yeast exhibited different nutritional needs than the fungi $A$. pullulans as the strain was not able to grow in the culture medium used for the FOS synthesis, neither in the initial conditions, containing sucrose in high concentration, nor in the final mixture containing mainly FOS and glucose. It was therefore necessary to optimize the broth composition ensuring the growth of the second strain. The fermentative broth was enriched with $5.0 \mathrm{~g} \mathrm{~L}^{-1}$ of yeast extract. In these conditions, $S$. cerevisiae was able to grow both in the initial medium and in the medium containing the FOS produced by $A$. pullulans and remaining sugars.

Since in co-culture, S. cerevisiae and A. pullulans are inoculated in the same medium, the influence of the yeast extract in the production of FOS by A. pullulans was first evaluated. Fig. 2 shows the time course of sucrose consumption and FOS production during the fermentations carried out in shaken flask (a) and bioreactor (b) with and without yeast extract. a)

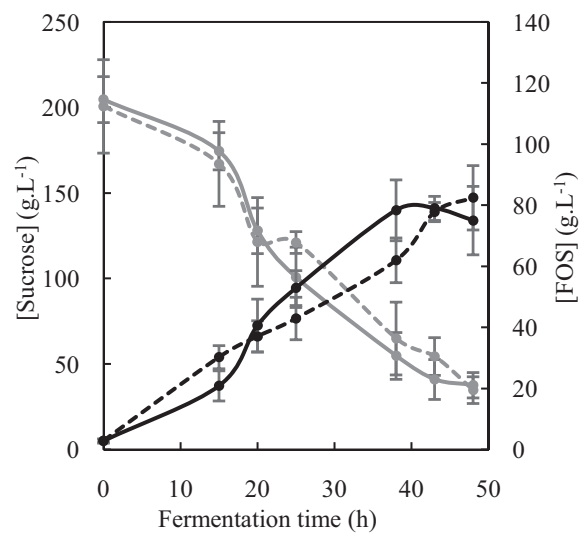

b)

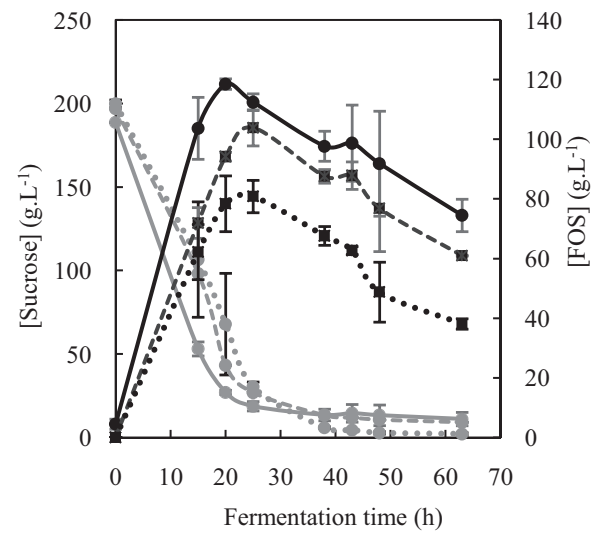

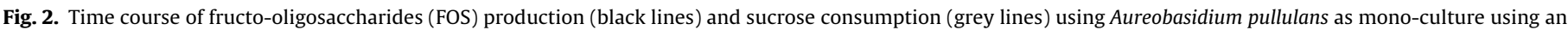

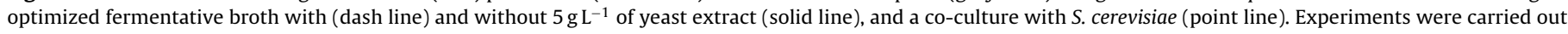
in shaken flask (a) and in bioreactor (b). 
Table 3

Fructo-oligosaccharides production in bioreactor using different fermentation strategies.

\begin{tabular}{|c|c|c|c|c|}
\hline Strategy & Microorganism & Fermentation time $(\mathrm{h})$ & FOS $\left(\mathrm{g} \mathrm{L}^{-1}\right)$ & $\%$ FOS $(w / w)^{a}$ \\
\hline Fermentation without yeast extract & Aureobasidium pullulans & 20 & $118.6 \pm 1.6$ & $54.0 \pm 1.6$ \\
\hline Fermentation with yeast extract & A. pullulans & 25 & $103.9 \pm 7.4$ & $53.8 \pm 1.0$ \\
\hline \multirow{2}{*}{ One-step fermentation } & \multirow[t]{2}{*}{ A. pullulans with Saccharomyces cerevisiae } & 25 & $80.8 \pm 5.4$ & $47.4 \pm 4.3$ \\
\hline & & 68 & $32.9 \pm 8.8$ & $60.9 \pm 0.4$ \\
\hline \multirow{2}{*}{ Two-step fermentation } & A. pullulans & 20 & $111.5 \pm 8.6$ & $51.2 \pm 1.7$ \\
\hline & S. cerevisiae & 68 & $100.6 \pm 6.1$ & $81.6 \pm 0.8$ \\
\hline
\end{tabular}

a On a dry weight basis.

The presence of yeast extract in this specific medium seems to delay the sucrose hydrolysis and decrease the productivity, especially in the assays run in bioreactor FOS $(p<0.05)$. In the bioreactor experiments, the yield decreased from $0.63 \pm 0.03$ to $0.53 \pm 0.04 \mathrm{~g}_{\mathrm{FOS}} \mathrm{g}_{\text {Sucrose }}{ }^{-1}$ and the maximum concentration of FOS produced was $104 \pm 6 \mathrm{~g} \mathrm{~L}^{-1}$. For the shaken flask experiments, the presence of yeast extract did not significantly affect the production of FOS ( $p \gg 0.05)$ (Fig. 2a).

Some studies have highlighted the negative impact of yeast extract on the production of the enzymes involved in the synthesis of several prebiotics, such as $\beta$-D-fructan-fructanohydrolase and inulin activity (Allais, Hoyos-Lopez, Kammoun, \& Baratti, 1987; Pandey et al., 1999). Indeed, (Ottoni et al., 2012) reported that increasing yeast extract concentrations above a given value had a negative effect on the $\beta$-fructofuranosidase production by Aspergillus oryzae IPT301. Nevertheless, the reasons for such effect have not yet been elucidated.

\subsection{Removal of the small saccharides from the fermentation broth}

S. cerevisiae can be conveniently used to remove mono- and disaccharides from the mixtures obtained from the enzymatic production of FOS. On the other hand, $\beta$-linked saccharides, such as 1-kestose, nystose and 1-fructofuranosyl nystose are not hydrolyzed by the yeast. Two strategies were evaluated to increase the percentage of FOS in relation to other sugars in the mixture: (1) one-step fermentation with both strains of A. pullulans and $S$. cerevisiae inoculated in co-culture in shaken flask/bioreactor, and (2) two-step fermentations consisting in the synthesis of FOS and further purification of the FOS mixture through the removal of the small saccharides by the yeast. Results obtained in each strategy plus the ones obtained from the conventional fermentation with $A$. pullulans with and without yeast extract in the fermentative broth are presented in Table 3.

\subsubsection{One-step fermentation}

In one-step fermentation, the fungus $A$. pullulans and the yeast $S$. cerevisiae were inoculated in the same fermentation vessel for simultaneous synthesis and purification of FOS. Results obtained from the co-culture fermentations in shaken flask and bioreactor are presented in Figs. 3 and 4, respectively.

The initial sucrose turnover rate decreased in the experiments conducted with $S$. cerevisiae in co-culture as compared to the fermentation run only with A. pullulans, without yeast extract supplementation (Fig. 2b). Nevertheless, the same sucrose concentration profile was obtained for fermentations run with yeast extract supplementation, both with A. pullulans solely and in coculture. Thus, this effect might be associated with the presence of some compounds from the yeast extract, such as amino acids and co-factors, rather than with the presence of $S$. cerevisiae.

In conventional fermentations carried out with $A$. pullulans, there is always some unreacted sucrose at the end of the fermentation due to inhibition, resulting from the presence of high amounts of glucose. In the present study, the sucrose amount

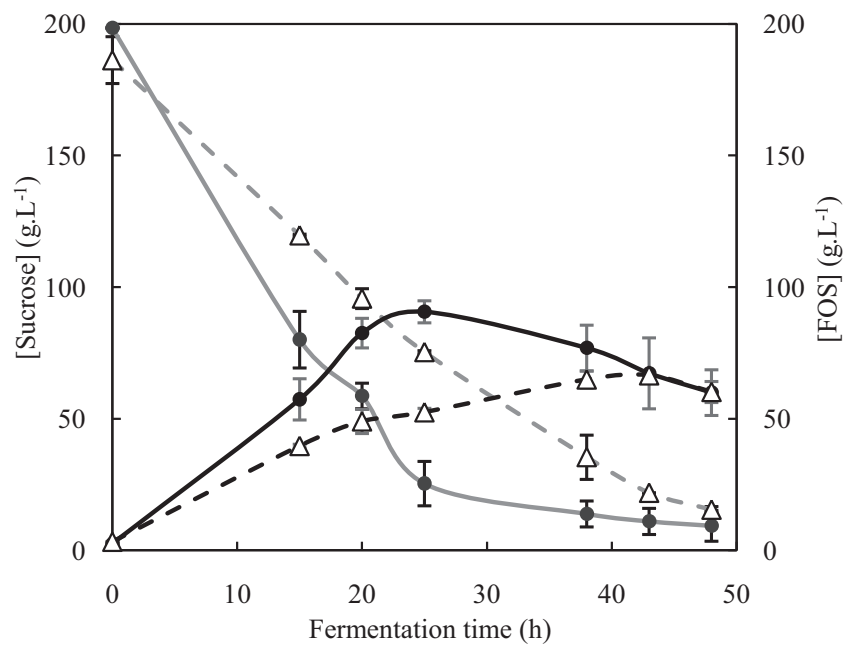

Fig. 3. Time course of fructo-oligosaccharides (FOS) production (black lines) and sucrose consumption (grey lines) using a mono-culture of Aureobasidium pullulans (solid line) and a co-culture with Saccharomyces cerevisiae (dash line). Experiments were carried out in triplicates using shaken flasks.

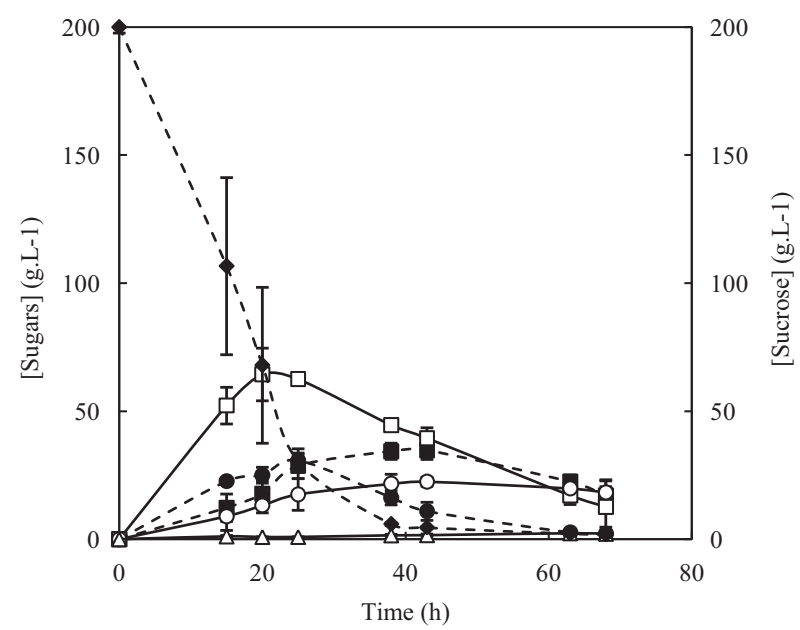

Fig. 4. Profile of sugars concentrations obtained during the co-culture fermentation carried out in bioreactor with $S$. cerevisiae and A. pullulans: $\mathbf{D}$ Fructose; Glucose; $\checkmark$ Sucrose; $\square \mathrm{GF}_{2} ; \bigcirc \mathrm{GF}_{3}$; and $\Delta \mathrm{GF}_{4}$.

contained in the fermentation broth at $20 \mathrm{~h}$ fermentation (time point at which FOS concentration was maximum), represented $12.3 \%$ of total sugars $\left(27.0 \mathrm{~g} \mathrm{~L}^{-1}\right.$ concentration). Even increasing the fermentation time to $63 \mathrm{~h}$, the amount of unreacted sucrose in the fermentation broth remained constant $\left(13.1 \pm 1.5 \mathrm{~g} \mathrm{~L}^{-1}\right)$. On the other hand, in the co-culture fermentation, the consumption rate of sucrose increased substantially after $25 \mathrm{~h}$ of fermentation and the sucrose amount decreased from $30.3 \mathrm{~g} \mathrm{~L}^{-1}$ (at $25 \mathrm{~h}$ of fermentation) to $1.7 \pm 1.1 \mathrm{~g} \mathrm{~L}^{-1}$ (at $68 \mathrm{~h}$ of fermentation), representing $3 \%$ of total sugars (Fig. 4 ). The decreased amount of sucrose in the 
co-culture fermentation broth might be related not only with the sucrose uptake by $S$. cerevisiae alone, but also with the recovery of the $\beta$-fructofuranosidase enzyme activity from the $A$. pullulans, to effectively transfer sucrose into FOS, as described by Yang et al. (2008).

In the experiments herein performed, using mono-culture fermentation in a bioreactor, $59.6 \mathrm{~g} \mathrm{~L}^{-1}$ of glucose were obtained in the fermentative broth at the time point corresponding to the maximum production of FOS, which accounted for $27.1 \%$ of the total sugars. In co-culture fermentation, the glucose concentration was found to be half of the amount for the same fermentation time, which accounted for $18.0 \%$ of the total sugars. By increasing the fermentation time, the glucose generated by the enzymatic synthesis of FOS was almost totally consumed by S. cerevisiae. On the other hand, fructose fermentation was much slower than glucose (Fig. 4). This might be explained by the glucophilic profile of $S$. cerevisiae. The yeast begins consuming fructose only when the glucose levels in the medium are low (Berthels, Otero, Bauer, Thevelein, \& Pretorius, 2004). Some glucose may also be transferred into fructose by glucose isomerase enzymes.

The transfructosylation reactions for the synthesis of FOS by $\beta$-fructotransferase, as well as the sucrose and FOS hydrolyzing reactions by $\beta$-fructofuranosidase, as proposed by Nishizawa et al. (2001) and Rocha et al. (2009) are described in Supplementary material.

1-Kestose concentration decreases with fermentation time due to its gradual conversion to nystose and simultaneous enzymatic hydrolysis (see Supplementary material Eqs. (A.2) and (A.8), respectively). In both mono and co-culture, the 1-kestose concentration profiles were similar. On the other hand, nystose and 1-fructofuranosyl nystose were formed in much lower concentrations in the co-culture fermentations.

Sucrose is hydrolyzed by invertase to glucose and fructose. Thus, it may be expected that $S$. cerevisiae competes for sucrose, thus reducing the amount of sucrose available to produce the second and third FOS, nystose and 1-fructofuranosyl nystose, respectively (see Supplementary material Eqs. (A.4) and (A.5)).

The maximum amount of FOS achieved in the co-culture fermentation, using a medium enriched with yeast extract, decreased from $103.9 \pm 6.1$ to $80.8 \pm 5.4 \mathrm{~g} \mathrm{~L}^{-1}$ in the presence of the $S$. cerevisiae strain as compared to fermentations run with A. pullulans alone (Table 3). Also, the yield of fermentation was lower $\left(0.40 \pm 0.03 g_{\text {FOS }} g_{\text {Sucrose }}{ }^{-1}\right)$, while the purity of FOS in the mixture was approximately the same. However, the content of FOS increased from the beginning to the end of fermentation since

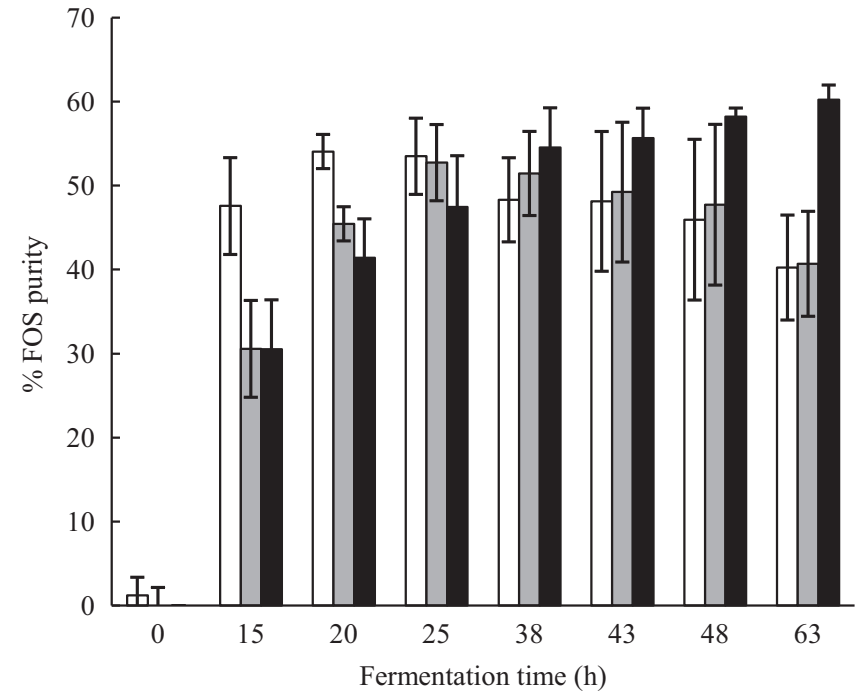

Fig. 5. Purity of fructo-oligosaccharides obtained during the fermentation in the bioreactor with: a mono-culture of Aureobasidium pullulans in an optimized fermentative broth with (grey bars) and without yeast extract (white bars); and a co-culture of A. pullulans and Saccharomyces cerevisiae (black bars). Experiments were carried out in triplicate.

sucrose and glucose were consumed by the strains. At $63 \mathrm{~h}$ of fermentation, a mixture containing 59.0\% purity of FOS was obtained instead of the $54.0 \%$ purity, usually obtained by fermentation with the single culture at $25 \mathrm{~h}$ of fermentation (point with maximum percentage of FOS) (Fig. 5).

An amount of $19.8 \mathrm{~g} \mathrm{~L}^{-1}$ of ethanol was produced from glucose through $S$. cerevisiae fermentation at the maximum FOS production time. The ethanol concentration increased to $72.4 \mathrm{~g} \mathrm{~L}^{-1}$ after $68 \mathrm{~h}$ of fermentation.

\subsubsection{Two-step fermentation}

The FOS synthesis with $A$. pullulans was stopped at $20 \mathrm{~h}$ of fermentation, i.e. the time point at which the maximum concentration of FOS was obtained in previous experiments conducted under optimized conditions. After removing the biomass corresponding to $A$. pullulans, a concentrated solution of yeast extract was added to the fermentative broth to enrich the nutritional composition of the medium, thus enabling the growth of S. cerevisiae.
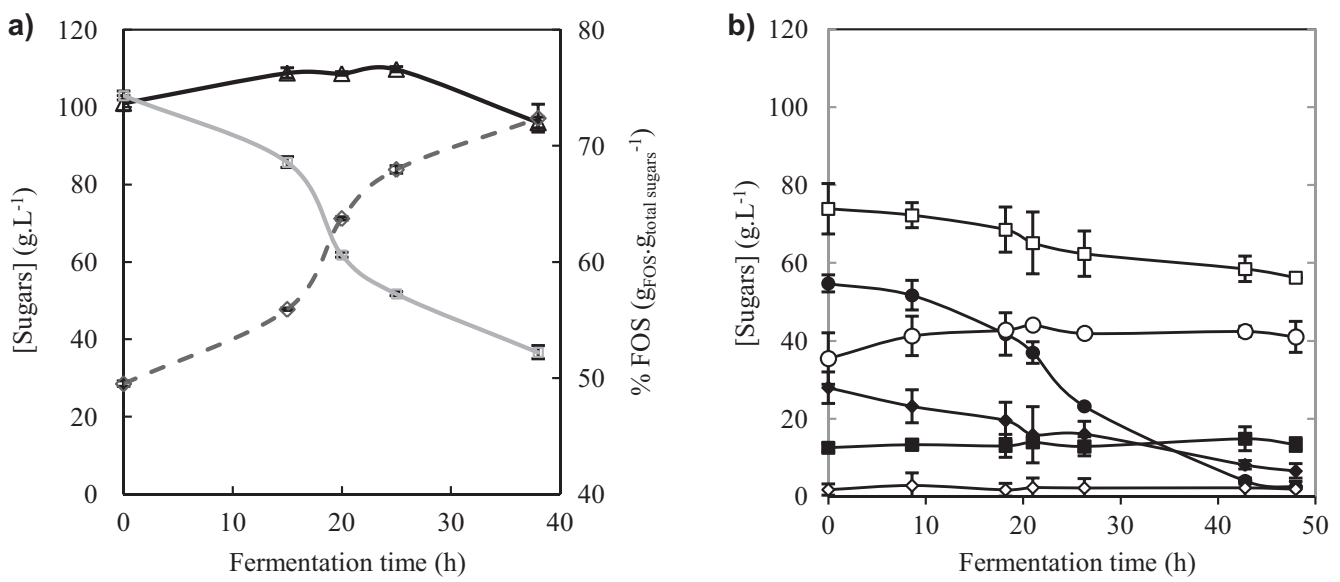

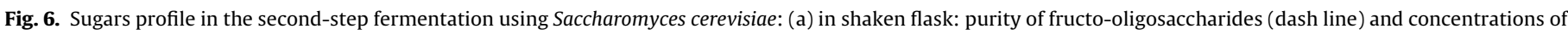
fructo-oligosaccharides (black full line) and sucrose (grey full line); (b) in bioreactor: $\square$ Fructose; $\bullet \mathrm{Glucose} \diamond$ Sucrose; $\square \mathrm{GF}_{2} ; \bigcirc \mathrm{GF}_{3} ;$ and $\diamond \mathrm{GF}_{4}$. 
Results obtained in shaken flasks for the second-step fermentation, with S. cerevisiae, are exhibited in Fig. 6a. S. cerevisiae presented the ability to remove small saccharides, as it can be observed by the increase of FOS percentage in total sugars, without lost of the total amount of FOS, until $20 \mathrm{~h}$ fermentation. After $20 \mathrm{~h}$, the FOS hydrolysis reactions rates were superior to the transfructolysation ones. The percentage of FOS in the mixture increased about $20 \%$ during the first $40 \mathrm{~h}$ of fermentation.

Fig. 6b presents the sugar profiles obtained for the secondstep fermentation, with S. cerevisiae, carried out in the bioreactor. Although the fermentative broth was filtrated to remove biomass from the fungi before the second-step fermentation, some enzymes released by $A$. pullulans kept their FOS synthesis activity and sucrose hydrolysis. Kestose concentration decreased during the second-step fermentation, from $73.9 \pm 4.7$ to $56.2 \pm 0.1 \mathrm{~g} \mathrm{~L}^{-1}$, while nystose concentration increased from $35.4 \pm 4.8$ to $41 \pm 2.9 \mathrm{~g} \mathrm{~L}^{-1}$, at $68 \mathrm{~h}$ fermentation. On the other hand, the glucose present in the fermentative broth was reduced after $S$. cerevisiae inoculation from $54.7 \pm 1.6 \mathrm{~g} \mathrm{~L}^{-1}$ to $2.4 \pm 0.1 \mathrm{~g} \mathrm{~L}^{-1}$, while the fructose concentration was kept constant around $13 \mathrm{~g} \mathrm{~L}^{-1}$, similarly to what was observed in the co-culture fermentation, thus showing again the preference of the yeast for glucose instead of fructose.

Through the fermentation of the mixture obtained from the first-step fermentation with $S$. cerevisiae, for $48 \mathrm{~h}$, the amount of total FOS decreased $10.0 \mathrm{~g} \mathrm{~L}^{-1}$ due to $\mathrm{GF}_{2}$ hydrolysis to monosaccharides (see Supplementary material Eq. (A.8)) and simultaneous $\mathrm{GF}_{3}$ synthesis (see Supplementary material Eq. (A.2)). This loss represents $9.0 \%$ of the total amount of FOS obtained in the first-step fermentation. However, by significantly decreasing the amount of glucose in the mixture, the purity of FOS increased substantially from $51.7 \%$ to $81.6 \%$.

In the current work, $48.1 \mathrm{~g} \mathrm{~L}^{-1}$ of ethanol were produced at the end of the second-step fermentation.

A similar approach as the one present in the current study was explored by Yang et al. (2008). The authors used Pichia pastoris to remove the excess of glucose converting it to glycerol. Their work was conducted with $\beta$-fructofuranosidase enzyme, for the production of FOS, and a successive fermentation with $P$. pastoris cells. FOS purity of the mixture increased from 56.55 to $84.45 \%$. The FOS yields reported are similar to the ones herein obtained. However, producing ethanol instead of glycerol seems to be advantageous since ethanol, as a volatile compound, is easily recovered by means of a reduced pressure distillation or a simple evaporation at moderate temperatures (Nobre et al., 2012).

The next step to obtain pure FOS mixtures (without small saccharides) is to use a SMB chromatography plant. Chromatography SMB columns generally separate sugars according to their molecular weight (Nobre, Teixeira, \& Rodrigues, 2015). Thus, sugars from the fermentative mixture would be desorbed in the following order: $\mathrm{GF}_{4}>\mathrm{GF}_{3}>\mathrm{GF}_{2}>\mathrm{GF}>\mathrm{G}>\mathrm{F}$. Sucrose is the nonoligosaccharide with closest retention time to FOS. Consequently, the peak of sucrose is the one that will overlap the oligosaccharides peaks (Nobre et al., 2014).

The final composition of the mixture obtained in terms of sugars for the two-step fermentation was: $10.9 \%$ fructose, $2.0 \%$ glucose, $5.4 \%$ sucrose, $46.3 \% \mathrm{GF}_{2}, 33.7 \% \mathrm{GF}_{3}$ and $1.6 \% \mathrm{GF}_{4}$. By decreasing the amount of sucrose from 27.9 to $6.6 \mathrm{~g} \mathrm{~L}^{-1}$ (from 13.5 to $5.4 \%$ of total sugars in the mixture), it is expected to obtain a better resolution in the chromatographic separation of oligo- from non-oligosaccharides. Therefore, the fermentation in series will contribute for the improved efficiency of the purification process of FOS by SMB, not only due to the increase of FOS content in the mixture, but also to the decreased amount of sucrose in the feed mixture.

\section{Conclusions}

The use of a microbial treatment prior to the SMB chromatography, was herein evaluated to pre-purify crude FOS mixtures. The fermentative broth salt composition was successfully significantly reduced without affecting the FOS production. Fermentations performed in a two-step process, i.e. using A. pullulans and S. cerevisiae sequentially, were more efficient in removing small saccharides present in the FOS mixtures than the co-culture fermentations. The second-step fermentation, conducted with S. cerevisiae alone, led to a mixture with $81.6 \%$ purity of FOS in total sugars, containing $5.4 \%$ sucrose. The final FOS mixture obtained in this work was enriched in FOS, but also contains low amounts of sucrose and salts which will greatly improve the efficiency of the next purification step, the SMB separation.

\section{Acknowledgements}

The financial support from the F.R.S.-FNRS, the Belgium National Fund for the Scientific Research, (PDR: T.0196.13) is gratefully acknowledged. Clarisse Nobre acknowledges to Fundação para a Ciência e a Tecnologia (Portugal) and POPH/FSE for the Post-Doc Grant received (reference SFRH/BPD/87498/2012). The authors acknowledge the financial support from the Strategic funding of UID/BIO/04469/2013 unit and project ref. RECI/BBBEBI/0179/2012 (project number FCOMP-01-0124-FEDER-027462) funded by Fundação para a Ciência e a Tecnologia.

\section{Appendix A. Supplementary data}

Supplementary data associated with this article can be found, in the online version, at doi:10.1016/j.carbpol.2015.08.088.

\section{References}

Allais, J. J., Hoyos-Lopez, G., Kammoun, S., \& Baratti, J. C. (1987). Isolation and characterization of thermophilic bacterial strains with inulinase activity. Applied and Environmental Microbiology, 53, 942-945.

Berthels, N. J., Otero, R. R. C., Bauer, F. F., Thevelein, J. M., \& Pretorius, I. S. (2004). Discrepancy in glucose and fructose utilisation during fermentation by Saccharomyces cerevisiae wine yeast strains. FEMS Yeast Research, 4, 683-689.

Crittenden, R. G., \& Playne, M. J. (2002). Purification of food-grade oligosaccharides using immobilised cells of Zymomonas mobilis. Applied Microbiology and Biotechnology, 58, 297-302.

De Preter, V., Hamer, H. M., Windey, K., \& Verbeke, K. (2011). The impact of preand/or probiotics on human colonic metabolism: Does it affect human health? Molecular Nutrition E' Food Research, 55, 46-57.

Dias, L. G., Veloso, A. C. A., Correia, D. M., Rocha, O., Torres, D., \& Rocha, I. (2009). UV spectrophotometry method for the monitoring of galacto-oligosaccharides production. Food Chemistry, 113, 246-252.

Dominguez, A., Nobre, C., Rodrigues, L. R., Peres, A. M., Torres, D., \& Rocha, I. (2012). New improved method for fructooligosaccharides production by Aureobasidium pullulans. Carbohydrate Polymers, 89, 1174-1179.

Haaland, P. D. (1989). Experimental design in biotechnology. New York: Marcel Dekker Inc.

Jung, K., Kim, J., Jeon, Y., \& Lee, J. (1993). Production of high fructo-oligosaccharide syrup with two enzyme system of fructosyltransferase and glucose oxidase. Biotechnology Letters, 15, 65-70.

Lin, T. J., \& Lee, Y. C. (2008). High-content fructooligosaccharides production using two immobilized microorganisms in an internal-loop airlift bioreactor. Journal of the Chinese Institute of Chemical Engineers, 39, 211-217.

Nishizawa, K., Nakajima, M., \& Nabetani, H. (2001). Kinetic study on transfructosylation by $\beta$-fructofuranosidase from Aspergillus niger ATCC 20611 and availability of a membrane reactor for fructooligosaccharide production. Food Science and Technology Research, 7, 39-44.

Nobre, C., Santos, M. J., Dominguez, A., Torres, D., Rocha, O., \& Peres, A. M. (2009) Comparison of adsorption equilibrium of fructose, glucose and sucrose on potassium gel-type and macroporous sodium ion-exchange resins. Analytica Chimica Acta, 654, 71-76.

Nobre, C., Suvarov, P., \& De Weireld, G. (2014). Evaluation of commercial resins for fructo-oligosaccharide separation. New Biotechnology, 31, 55-63.

Nobre, C., Teixeira, J. A., \& Rodrigues, L. R. (2012). Fructo-oligosaccharides purification from a fermentative broth using an activated charcoal column. New Biotechnology, 29, 395-401. 
Nobre, C., Teixeira, J. A., \& Rodrigues, L. R. (2015). New trends and technological challenges in the industrial production and purification of fructo-oligosaccharides. Critical Reviews in Food Science and Nutrition, 55, 1444-1455.

Ottoni, C. A., Cuervo-Fernández, R., Piccoli, R. M., Moreira, R., Guilarte-Maresma, B., da Silva, E. S., et al. (2012). Media optimization for $\beta$-fructofuranosidase production by Aspergillus oryzae. Brazilian Journal of Chemical Engineering, 29, 49-59.

Pandey, A., Soccol, C. R., Selvakumar, P., Soccol, V. T., Krieger, N., \& Fontana, J. D. (1999). Recent developments in microbial inulinases - Its production, properties, and industrial applications. Applied Biochemistry and Biotechnology, 81, 35-52.

Pinelo, M., Jonsson, G., \& Meyer, A. S. (2009). Membrane technology for purification of enzymatically produced oligosaccharides: Molecular and operational features affecting performance. Separation and Purification Technology, 70, $1-11$.

Rocha, O., Nobre, C., Dominguez, A., Torres, D., Faria, N., \& Rodrigues, L. R. (2009). A dynamical model for the fermentative production of fructooligosaccharides. Computer Aided Chemical Engineering, 27, 1827-1832.

Sangeetha, P. T., Ramesh, M. N., \& Prapulla, S. G. (2005). Recent trends in the microbial production, analysis and application of fructooligosaccharides. Trends in Food Science \& Technology, 16, 442-457.

Sheu, D. C., Chang, J. Y., Wang, C. Y., Wu, C. T., \& Huang, C. J. (2013). Continuous production of high-purity fructooligosaccharides and ethanol by immobilized
Aspergillus japonicus and Pichia heimii. Bioprocess and Biosystems Engineering, 36, 1745-1751.

Sheu, D. C., Duan, K. J., Cheng, C. Y., Bi, J. L., \& Chen, J. Y. (2002). Continuous production of high-content fructooligosaccharides by a complex cell system. Biotechnology Progress, 18, 1282-1286.

Sheu, D. C., Lio, P. J., Chen, S. T., Lin, C. T., \& Duan, K. J. (2001). Production of fructooligosaccharides in high yield using a mixed enzyme system of $\beta$-fructofuranosidase and glucose oxidase. Biotechnology Letters, 23, 1499-1503.

Yang, Y.-L., Wang, J.-H., Teng, D., \& Zhang, F. (2008). Preparation of high-purity fructo-oligosaccharides by Aspergillus japonicus $\beta$-fructofuranosidase and successive cultivation with yeast. Journal of Agricultural and Food Chemistry, 56, 2805-2809,

Yoshikawa, J., Amachi, S., Shinoyama, H., \& Fujii, T. (2008). Production of fructooligosaccharides by crude enzyme preparations of $\beta$-fructofuranosidase from Aureobasidium pullulans. Biotechnology Letters, 30, 535-539.

Yun, J. W., \& Song, S. K. (1993). The production of high-content fructo-oligosaccharides from sucrose by the mixed-enzyme system of fructosyltransferase and glucose-oxidase. Biotechnology Letters, 15, 573576.

Yun, J. W., Lee, M. G., \& Song, S. K. (1994). Batch production of high-content fructo-oligosaccharides from sucrose by the mixed-enzyme system of $\beta$-fructofuranosidase and glucose oxidase. Journal of Fermentation and Bioengineering, 77, 159-163. 